\title{
The Role of MicroRNAs in Response to Interferon Treatment of Chronic Hepatitis $\mathbf{C}$ patients
}

Eman El-Ahwany ${ }^{1}$, Faten Nagy ${ }^{1}$, Mona Zoheiry ${ }^{1}$, Maged ELGhannam², Mohamed Shemis ${ }^{3}$, Mohamed AboulEzz $^{2}$, Suher Zada ${ }^{4}$

${ }^{1}$ Immunology Department, Theodor Bilharz Research Institute, Giza, Egypt

${ }^{2}$ Hepato-gastroenterology Department, Theodor Bilharz Research Institute, Giza, Egypt

${ }^{3}$ Biochemistry Departm, Theodor Bilharz Research Institute, Giza, Egypt

${ }^{4}$ Biology Department, the American University in Cairo, Egypt

\section{Type of article: Original}

\begin{abstract}
Introduction: Treatment of HCV using a combination of pegylated interferon (PEG-IFN) and ribavirin fails in about $40 \%$ of the patients with HCV genotype 4 infections, and it is physically and economically demanding. Thus, it is highly important to identify factors that can help to predict the likelihood that a patient will respond to this treatment.

Methods: In this study, five miRNAs, i.e., miRNA-122, miRNA-199, miRNA-192, miRNA-30, and miRNA128 , were selected according to previous studies that demonstrated their noticeable functions in viral replication, indicating that they potentially could be used by host cells to control viral infections. The five miRNAs were measured using real-time, reverse transcription-polymerase chain reactions. The data were analyzed using the $\mathrm{t}$ test and chi-squared test.

Results: We found that the expression level of miRNA-122 was significantly increased in the responders' group $(\mathrm{p}<0.01)$ over that in the non-responders' groups before and after treatment; both increased significantly ( $<$ 0.01 ) compared with the normal control group.

Conclusion: miR-122 might be a useful predictor for virological responses to treatment with PEG-interferon plus ribavirin therapy in patients with $\mathrm{HCV}$.

Keywords: microRNA, Interferon, Chronic Hepatitis C, HCV, pegylated interferon
\end{abstract}

\section{Introduction}

Hepatitis $\mathrm{C}$ virus $(\mathrm{HCV})$ is a highly persistent human pathogen. It has been estimated that 180 million people are infected by this pathogen worldwide, and it can cause chronic liver disease that progresses to liver failure and/or cancer (1). Treatment of patients with chronic hepatitis $\mathrm{C}$ with pegylated interferon (PEG-IFN) and ribavirin achieves overall sustained virologic response rates of approximately $55 \%$ (2). Recently, strategies for treating HCV infection have been improved by newly discovered direct antiviral agents (DAAs), which inhibit viral enzymes (e.g., protease and polymerase inhibitors) $(1,3)$. The current standard therapy for CHC is triple therapy with pegylated IFN, ribavirin, and one of the HCV protease or polymerase inhibitors. Therefore, even in the presence of DAAs, interferon (IFN) still will remain as the backbone for the combination therapy. This is due to the fact that viralresistant mutants emerge rapidly if DAA is used alone (3). MicroRNAs are small, endogenous, non-coding, 22 nucleotide-long RNAs that regulate gene expression at post transcription of the mRNA or suppression of protein translation upon binding to the 3'untranslated regions (UTRs) of an mRNA (4-6). The expression of miRNAs is strictly limited to specific organs and tissues. MiRNA-122, for example, is only abundantly expressed in the liver and has not been detected in other tissues (7-9). Also, progression of hepatic fibrosis was shown to be significantly correlated with over-expression of miRNA-199 (10). Previous studies also demonstrated that miRNAs levels differ depending on the patient's response to the drug. So, due to the fact that almost of half of the patients fail to respond

\section{Corresponding author:}

Eman El-Ahwany, Immunology Department, Theodor Bilharz Research Institute, Giza, Egypt.

E-mail: Ahwany@aucegypt.edu

Received: December 01, 2015, Accepted: January 24, 2016, Published: February 2016

iThenticate screening: January 03, 2016, English editing: January 28, 2016, Quality control: February 02, 2016

(C) 2016 The Authors. This is an open access article under the terms of the Creative Commons Attribution-NonCommercialNoDerivs License, which permits use and distribution in any medium, provided the original work is properly cited, the use is non-commercial and no modifications or adaptations are made. 
to IFN-based therapy, patients would benefit from a prognostic tool to predict the likelihood of a response to the treatment prior to the administration of the combination therapy, or at least soon after beginning the therapy, thereby reducing the incidence of ineffective treatments $(3,11)$. Changes in circulating miRNA profiles may be considered potential biomarkers because of their stability in body fluids, sensitive detection by quantitative PCR, and noninvasiveness in obtaining samples of body fluid (12). In this study, we selected five miRNAs, i.e., miRNA-122a, miRNA-199, miRNA-192, miRNA-30, and miRNA-128, to investigate their levels in the serum of patients with CHC treated with PEG-IFN and ribavirin to determine the role of miRNAs in association with the drug response and to hence to predict the likelihood of a positive response to the treatment prior to the initiation of the therapy.

\section{Material and Methods}

\subsection{Study Design}

This study was conducted on patients with hepatitis $\mathrm{C}$ virus infection coming to the out-patient clinic of HepatoGastroenterology at Theodor Bilharz Research Institute (TBRI). The local institutional review board at the Institute approved this study, and all individuals gave written informed consent according to the ethical guidelines of the 1975 Declaration of Helsinki. None of them was co-infected with HIV or hepatitis B virus (HBV). The study included 60 patients who had chronic HCV infection and were eligible for interferon-based treatment plus ribavirin therapy. Of the 60 patients, 25 were responders (one half year after negativity of HCV-RNA-PCR after end of treatment), and 35 were non-responders (persistence of HCV-RNA-PCR in spite of IFN therapy for at least three months). The control group consisted of 20 healthy people, and blood samples were collected before and after treatment.

\subsection{Laboratory investigations}

We conducted liver function tests and serologic diagnosis of viral hepatitis to determine whether the patients had chronic HCV infections. It was determined that the cases included in the study had reactive anti-HCV antibodies for more than six months (using Murex enzyme immunoassay kit, Dartford, England); a positive HCV-RNA by PCR; absence of other CLD as hepatitis B (defined as negative reaction to HBV surface antigen and HBV core antibody), autoimmune hepatitis (negative reaction to anti-nuclear, anti-smooth muscle, anti-mitochondrial and anti-liverkidney microsomal antibodies), schistosomiasis mansoni (no previous history and negative stool examination); no previous history of regular use of hepato-toxic drugs or alcohol abuse. We also excluded patients who had been treated earlier for hepatitis C. Past medical records, pathology reports, and personal interviews were used to acquire data on all the subjects. We collected extensive data, including age, gender, serum albumin (ALB) level, total bilirubin level (T-Bil), and alanine aminotransferase (ALT) level.

\subsection{Methods}

All patients received 24 weeks of antiviral therapy consisting of orally administered ribavirin and subcutaneous infusion with pegylated IFN- $\alpha$, both of which were weight-based. All of the responders completed 48 weeks of treatment. However, the non-responders stopped the treatment at 24 weeks. So, for comparative reasons, analyses for both groups were conducted after 24 weeks of treatment. The diagnosis of HCV infection was based on clinical features, laboratory tests, and liver biopsies. Full medical history, clinical examination, laboratory investigations, and abdominal ultrasonography were performed for all patients at the beginning of treatment. Blood and plasma samples were collected at the start of treatment ( 0 day) and at 12 and 24 weeks of treatment. According to the response of the HCV patients to peg IFN $\alpha$ plus ribavirin therapy, the patients were classified into two groups, and 32 patients were responders to treatment (sustained virologic response) with PCR for HCV RNA was negative after three months of treatment and also six months after the treatment had ended. Twenty-seven patients were nonresponders to the treatment (non-sustained virological response) with persistence of positivity of HCV- RNA- PCR after three months of treatment.

\subsection{Serum Preparation and RNA Extraction}

The blood samples were centrifuged at $1600 \mathrm{rpm}$ for $5 \mathrm{~min}$, and the serum was aliquoted into 1.7-ml Eppendorf tubes. This was followed by high-speed centrifugation at 12,000 rpm for 15 minutes to completely remove cell debris and leave only circulating RNA. In order to isolate the RNA from the serum, $250 \mu \mathrm{l}$ of the serum were homogenized in $750 \mu \mathrm{l}$ of Trizol LS (Invitrogen). Then, $200 \mu \mathrm{l}$ of chloroform were added to the sample, and the solution was mixed and then centrifuged. After another chloroform extraction followed by precipitation with isopropanol, the pellet was washed twice by centrifugation with $70 \%$ ethanol. Then, the pellet was dried for $10 \mathrm{~min}$ at room temperature and dissolved in $30 \mu \mathrm{l}$ of diethylpyrocarbonate (DEPC)-treated water. DNase treatment (Qiagen) was conducted to remove any DNA that could contaminate the sample. The concentration and quality of 
RNA were measured by UV absorbance at $260 \mathrm{~nm}$ and $280 \mathrm{~nm}$ (A260/280 ratio), and they were individually checked by gel electrophoresis. In this way, $1 \mathrm{ml}$ of serum was used to produce $600 \mathrm{ng}$ of RNA.

\subsection{Assessment of HCV-4 RNA loads by Real-time PCR}

After RNA was extracted from the serum using a Qiagen Viral RNA Kit (Hilden, Germany), viral loads were measured by the real-time, reverse transcription polymerase chain reaction (qRT-PCR) using a light Cycler system (Roche Diagnostics GmbH, Mannheim, Germany). Amplification primers for $\mathrm{HCV}$ were $5^{`}$ primer $\mathrm{K} 78 \mathrm{~F}$ (CAAGCACCCTATCAGGCAGT) and 3 'primer K80R (AGCGTCTAGCCATGGCGT). The product was detected by $3^{\prime}$ and LC5 (CCCGGGAGAGCCATAGTGGTCTG) 3`. Before treatment, the HCV-RNA in the serum was measured, and it was re-measured routinely at 24 and 48 weeks after treatment. The results of the measurements were graded as low, moderate, and high levels.

\subsection{Reverse Transcription (RT) and Quantitative PCR ( $q P C R$ )}

RT and qPCR kits made specifically for accurate miRNA analysis (Applied Biosystems) were used to evaluate expression of the chosen miRNAs, i.e., miR-122, miR-199, miR-192, miR-30, and miR-128, from the serum samples. The miRNA-16 was used as the endogenous control. The 15- $\mu \mathrm{L}$ RT reactions were performed using a TaqMan ${ }^{\circledR}$ microRNA Reverse Transcription Kit (Applied Biosystems, USA), incubated for 30 min at $16{ }^{\circ} \mathrm{C}, 30$ min at $42{ }^{\circ} \mathrm{C}$, and $5 \mathrm{~min}$ at $85^{\circ} \mathrm{C}$. Then they were maintained at $4{ }^{\circ} \mathrm{C}$. For real-time PCR, $1.33 \mu \mathrm{L}$ of the diluted RT products were mixed with $10 \mu \mathrm{L}$ of $2 \times$ Taqman PCR master mixture (No AmpErase UNG), $1 \mu \mathrm{L}$ of the TaqMan MicroRNA Assay, and $7.67 \mu \mathrm{L}$ of the Nuclease-free water, making a final volume of $20 \mu \mathrm{L}$ based on the manufacturer's instructions. All reactions were run on the ABI 7300 (Applied Biosystems, USA) using the following conditions: $95{ }^{\circ} \mathrm{C}$ for $10 \mathrm{~min}$, followed by 40 cycles at $95{ }^{\circ} \mathrm{C}$ for $15 \mathrm{~s}$, and $60{ }^{\circ} \mathrm{C}$ for $1 \mathrm{~min}$. Real-time PCR was done in triplicate, including no-template controls. Relative expression of miRNA was calculated using the comparative cycle threshold (CT) $\left(2^{-\Delta \Delta C T}\right)$ method with miRNA-16 as the endogenous control to normalize the data. CT was defined as the number of cycles required for the FAM signal to cross the threshold in the real-time PCR. $\Delta \mathrm{CT}$ was calculated by subtracting the $\mathrm{CT}$ values of miR-16 from the $\mathrm{CT}$ values of the chosen miRNA. Then, $\Delta \Delta \mathrm{CT}$ was calculated by subtracting the mean $\Delta \mathrm{CT}$ of the control samples from the $\Delta \mathrm{CT}$ of the tested samples. Fold change of miRNA was calculated by equation $2^{-\Delta \Delta C T}$.

\subsection{Statistical analysis}

The data were expressed as mean \pm standard error or percentage (\%). Comparison between the mean values of different parameters in the two groups was performed using the unpaired t-test. Comparison between categorical data was performed using the chi-squared test. The SPSS software program was used to analyze the data, and pvalues less than or equal to 0.05 were considered significant; values $<0.01$ were considered to be highly significant.

\section{Results}

The laboratory investigation showed that there was a highly significant difference in the non-responders group compared to the responders group $(\mathrm{p}<0.01)$ in terms of ALT and AST activities. However, the other biochemical findings showed no significant difference between the two groups. Laboratory data of the cases are presented in Table 1.

Table 1. Biochemical findings measured before and during treatment for all patients

\begin{tabular}{|l|l|l|l|}
\hline Variables & $\mathrm{NR}(\mathrm{n}=25)$ & $\mathrm{R}(\mathrm{n}=25)$ & $\mathrm{p}$-value \\
\hline ALT (U/L) & $47.64 \pm 28.71$ & $23.16 \pm 11.60$ & 0.001 \\
\hline AST (U/L) & $47.76 \pm 30.21$ & $23.04 \pm 10.59$ & 0.001 \\
\hline Total Protein $(\mathrm{g} / \mathrm{dL})$ & $7.79 \pm 0.48$ & $7.73 \pm 0.57$ & 0.689 \\
\hline Albumin $(\mathrm{g} / \mathrm{dL})$ & $4.06 \pm 0.46$ & $3.94 \pm 0.33$ & 0.290 \\
\hline Total bilirubin(mg/dL) & $0.95 \pm 0.85$ & $0.84 \pm 0.38$ & 0.550 \\
\hline Direct bilirubin(mg/dL) & $0.32 \pm 0.30$ & $0.41 \pm 0.22$ & 0.220 \\
\hline AFP $(\mathrm{U} / \mathrm{ml})$ & $7.80 \pm 11.69$ & $6.78 \pm 4.89$ & 0.687 \\
\hline Createnin (mg/dL) & $0.91 \pm 0.13$ & $1.01 \pm 0.26$ & 0.105 \\
\hline Urea $(\mathrm{mg} / \mathrm{dL})$ & $25.44 \pm 7.74$ & $25.96 \pm 9.07$ & 0.828 \\
\hline
\end{tabular}

Data are expressed as mean $\pm \mathrm{SD}$, ALT: Alanine amino transferase, AST: Aspartate amino transferase, AFP: Alpha feto protein, Normal range for albumin is 3.5-5 g/dL, Normal range for ALT and AST is up to 40 UmL, Normal range for AFP is $<$ or $=20 \mathrm{U} / \mathrm{ml}$. 
MicroRNAs expression level in patients before treatment showed significant increase in expression of miR-122 in the responders group $(p<0.01)$ with no significant difference in the other miRNAs between the two groups (Figure 1). MicoRNAs expression levels in patients after 12 weeks of treatment with PEG-IFN/RBV showed a significant increase in expression of miR-122 in the responders group $(\mathrm{p}<0.01)$ and a significant increase in miRNAs in the responders and non-responders groups compared to before treatment $(\mathrm{p}<0.01)$ (Figure 2$)$.

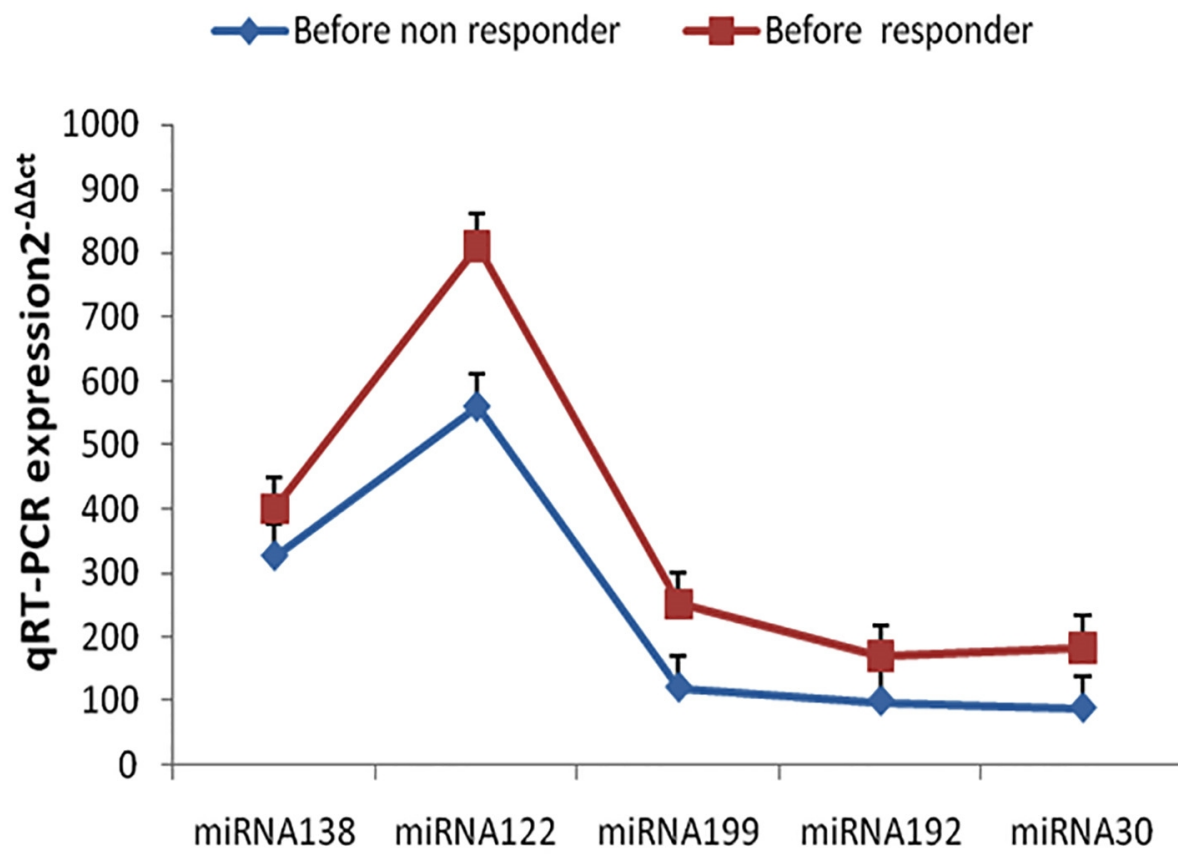

Figure 1. Real time qPCR of miRNAs expression levels in responders and non-responders groups before treatment.
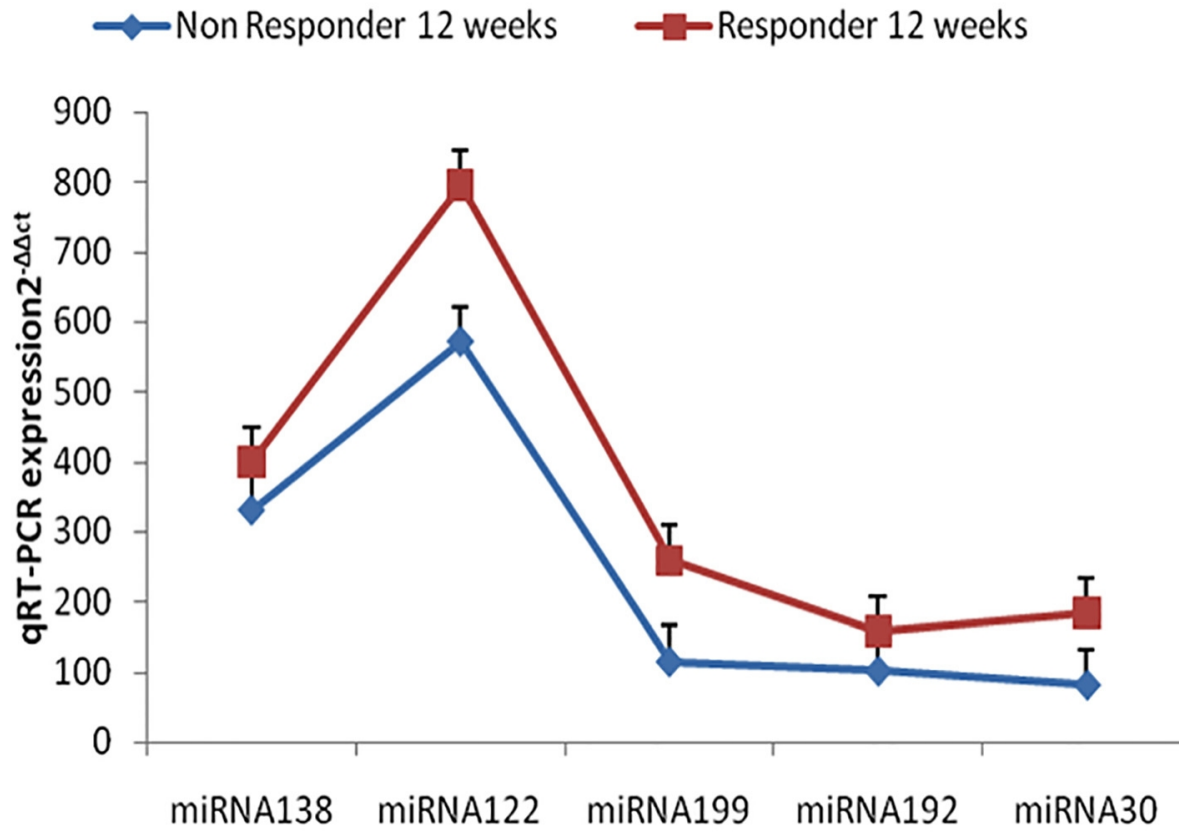

Figure 2. Real time qPCR of miRNAs expression levels in responders and non responders groups after 12 weeks treatment.

The other miRNAs expression showed no significant difference between responders and non-responders groups. MiRNAs expression levels in patients after 24 weeks of treatment showed a significant increase in miR-122 in 
responders group $(p<0.01)$ compared to the non-responders group $(p<0.01)$ and compared to the before treatment group. However, the expression of the other miRNAs showed no significant difference between the responder and non-responder groups (Figure 3).

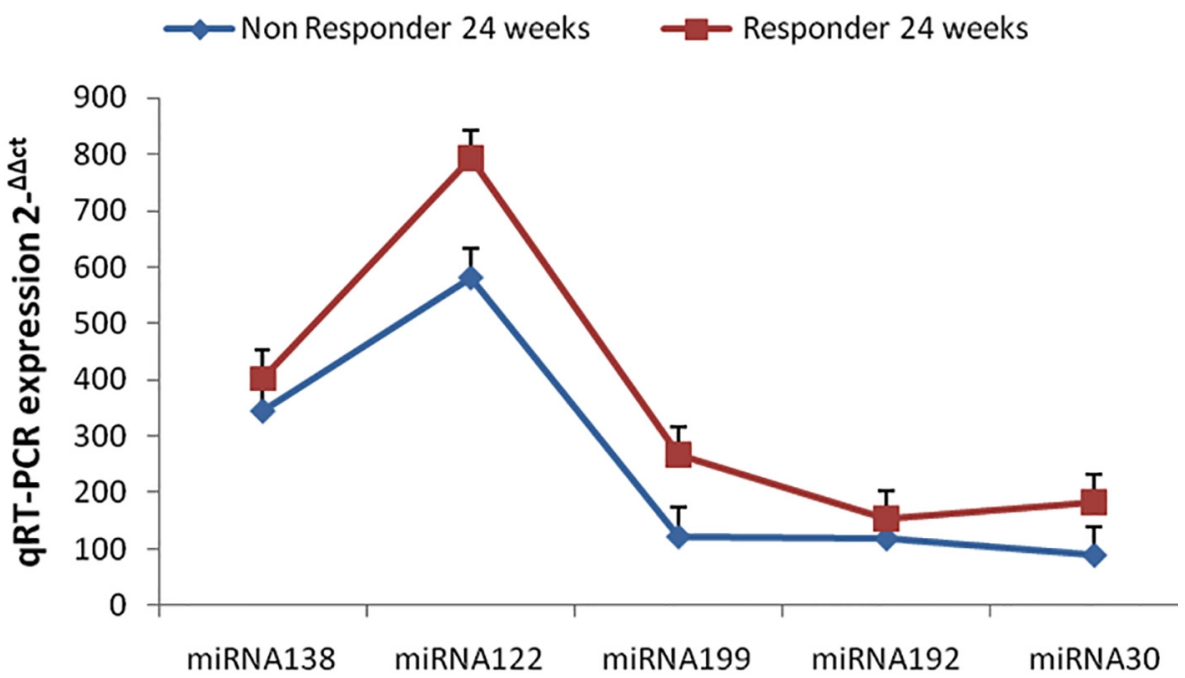

Figure 3. Real-time qPCR of miRNAs expression levels in responders and non-responders groups after 24 weeks of treatment

\section{Discussion and Conclusions}

Current standard IFN-RBV therapy against HCV is known to be effective in only 50\% of infected patients (6). Recently, DAAs were added to PEG-IFN/RBV, leading to higher sustained response, considering that the trace amount of HCV RNA from successfully treated patients can be infectious $(1,6)$. Several miRNAs participated in the control of HCV infection and are differently regulated by interferon treatment, suggesting a different host response to both HCV and IFN-treatment $(6,8,13,14)$. In this study, we measured the expression of five miRNAs, i.e., miR122, miR-199, miR-192, miR-128, and miR-30, in the serum of HCV patients who responded or did not respond to PEG-IFN/RBV treatment 12 and 24 weeks after the beginning of the treatment. Previous studies confirmed that the expression of these miRNAs is altered upon treatment with interferon and, at the same time, they directly modulate the IFN-mediated antiviral response (13-16). MiR-122 represents the most abundant liver-miRNA, and it has an anti-inflammatory role in the liver (16). The serum level of miR-122 has a positive correlation with hepatic miR-122 expression (17). Also, miR-128 and miR-199 were expressed in hepatitis C-derived myofibroblasts (18). MiR-199 has been reported to interact directly with $\mathrm{HCV}$ genome and upon binding to $\mathrm{HCV}$ genome and upon binding to HCV RNA, it inhibits HCV replication (1, 19, 20). The inhibition of miR-30 increased the level of HCV RNA by creating a permissive environment of viral replication and this "pro-viral effect is diminished with addition of IFN- $\alpha$ as a treatment (14). In contrast, it was observed that miR-192 is capable of enhancing HCV replication, and it was observed in the serum of patients with chronic hepatitis $\mathrm{C}(20)$.

This study demonstrated that liver-specific micro RNA miR-122 was up-regulated in the responders group after 12 and 24 weeks of treatment, and it also was highly expressed in the responders group even before treatment. However, other miRNAs (miR-138, miR-192, miR-199, miR-30) showed no significant difference in the responders and non-responders groups after treatment or before treatment. Previous studies has shown that expression levels of these miRNAs were significantly different in the responders and non-responders groups, suggesting that the expression pattern of these hepatic miRNA was associated with therapeutic outcome in the treated patients (13, 20). The expression level of miR-122 was reportedly associated with early response to IFN treatment, and it was found to be markedly decreased to pre-treatment miR-122 levels in patients who had no virological response during IFN therapy $(3,6,11,13,17,20-22)$. A recent study found that interferon stimulated gene (ISG) is an miR-122 target and reduced induction of ISG during peg IFN/RBV treatment was found to be associated with poor virologic responses $(17,21)$. It also was hypothesized that the non-responders may have a higher expression of ISG, which down-regulates hepatic miR-122 and thus leads to a low level of serum miR-122 (17, 21). Another study demonstrated that miR-122 is known to be a positive cofactor in HCV replication (11) and within the UTRs of HCV 
RNA, three highly conserved miR-122 recognition elements exist with two in the 5'UTR and one in the 3 'UTR. The 3'UTR site appears to have no role in the stimulation of viral replication or translation (23). So, it may be during treatment with IFN/RBV responders' patients, miR-122 bind to 3'UTR which impairs HCV RNA replication and translation $(20,23)$. Our conclusion is in agreement with $\mathrm{Su}$ et al. (17) and Motawi et al. (11), who suggested that higher pretreatment serum miR-122 levels might predict favorable virological responses to PEG-IFN/RBV therapy in patients with $\mathrm{HCV}$.

\section{Acknowledgments:}

The authors sincerely thank the staff at the Clinic of Hepato-Gastroenterology (Theodor Bilharz Research Institute) for their help in this study.

\section{Conflict of Interest:}

There is no conflict of interest to be declared.

Authors' contributions:

All authors contributed to this project and article equally. All authors read and approved the final manuscript.

\section{References}

1) Kwon YC, Ray RB, Ray R. Hepatitis C virus infection: establishment of chronicity and liver disease progression. EXCLI J. 2014; 13: 977-96. PMID: 26417315, PMCID: PMC4464452.

2) Asselah T, Benhamou Y, Marcellin P. Protease and polymerase inhibitors for the treatment of hepatitis $C$. Liver Int. 2009; 29: 57-67. doi: 10.1111/j.1478-3231.2008.01928.x, PMID: 19207967.

3) Yuan Li, Yuan J, Yujia Li, Shilin Li, Duan X, Liu B, et al. Host factors to predict treatment response in $\mathrm{HCV}$ patients: Implications for individualized therapy and translational medicine for HCV. J Bioanal Biomed. 2013; 5: 4-6.

4) Davidson-Moncada J, Papavasilion FN, Tam W. Micro RNAs of the immune system: roles of inflammation and cancer. Ann N Y Acad Sci. 2010; 1183: 183-94. doi: 10.1111/j.1749-6632.2009.05121.x, PMID: 20146715, PMCID: PMC2876712.

5) Liu X, Wang T, Wakita T, Yang W. Systematic identification of micro RNA and messenger RNA profiles in hepatitis C virus-infected human hepatoma cells. Virology. 2010; 398: 57-67. doi: 10.1016/j.virol.2009.11.036, PMID: 20006370.

6) Gelley F, Zadori G, Nemes B, Fassan M, lendvai G, Sarvary E, et al. Micro RNA profile before and after antiviral therapy in liver transplant recipients for hepatitis C virus cirrhosis. J Gastroenterol Hepatol. 2014; 29: 121-7. doi: 10.1111/jgh.12362, PMID: 24033414.

7) Lagos-Quintana M, Rauhut R, Yalcin A, Meyer J, Lendeckel W, Tuschl T. Identification of tissue-specific micrornas from mouse. Curr Biol. 2002; 12: 735-9. doi: 10.1016/S0960-9822(02)00809-6.

8) Neilson JR, Zheng GX, Burge CB, Sharp PA. Dynamic regulation of miRNA expression in ordered stages of cellular development. Genes Dev. 2007; 21: 578-89. doi: 10.1101/gad.1522907, PMID: 17344418, PMCID: PMC1820899.

9) Berkhout B. A balancing act: viruses and miRNAs. J Formos Med Assoc. 2008; 107: 1-3. doi: 10.1016/S0929-6646(08)60001-7.

10) Haybaeck J, Zeller N, Heikenwalder M. The parallel universe: micro RNAs and their role in chronic hepatitis, liver tissue damage and hepatocarcinogenesis. European journal of medical sciences. 2011; 141: 13287-90.

11) Motawi TM, Rizk SM, Shaker OG, Mokhtar OZH. Micro RNAs predictor markers for response to interferon treatment of chronic hepatitis C genotype-4 in Egyptian patients. PLoS ONE. 2015; 10(3): 137193.

12) Hoffmann TW, Gilles D, Bengrine A. Micro RNAs and hepatitis $C$ virus: Toward the end of miR-122 supremacy. Virol J. 2012; 9: 109-16. doi: 10.1186/1743-422X-9-109, PMID: 22691570, PMCID: PMC3489824.

13) Murakami Y, Tanaka M, Toyoda H, Hayashi K, Kuroda M, Tajima A, et al. Hepatic micro RNA expression is associated with the response to interferon treatment of chronic hepatitis C. BMC Med Genomics. 2010; 3: 48-57. doi: 10.1186/1755-8794-3-48, PMID: 20969775 , PMCID: PMC2984584.

14) Zhang X, Daucher M, Armistead D, russel R, Kottilil Sh. Micro RNA expression profiling in HCV-infected human hepatoma cells identifies potential anti-viral targets induced by interferon- $\alpha$. Plos One. 2013; 8(2): e55733. doi: 10.1371/journal.pone.0055733, PMID: 23418453, PMCID: PMC3572124. 
15) Shwetha S, Gouthamchandra K, Chandra M, Ravishankar B, Khaja M, Das S. Circulating miRNA profile in HCV infected serum: novel insight into pathogenesis. Scientific Reports. 2013; (3): 1-7. doi: 10.1038/srep01555.

16) Roy S, Benz F, Ludde T, Roderburg C. The role of miRNAs in the regulation of inflammatory processes during hepatofibrogenesis. Hepatobiliary Surg Nutr. 2015; 4(1): 24-33. doi: 10.3978/j.issn.23043881.2015.01.05, PMID: 25713802, PMCID: PMC4318955.

17) $\mathrm{Su} \mathrm{T}$, Liu $\mathrm{CH}$, Liu CJ, Chen C, Ting $\mathrm{T}$, Tseng $\mathrm{T}$, et al. Serum microRNA-122 level correlates with Virologic responses to pegylated interferon therapy in chronic hepatitis C. proc Natl Acad Sci. 2013; 110(19): 7844-9. doi: 10.1073/pnas.1306138110, PMID: 23613588, PMCID: PMC3651447.

18) Page A, Mann D, Mann J. The mechanisms of HSC activation and epigenetic regulation of HSCs phenotypes. Curr Pathobiol Res. 2014; (2): 163-70. doi: 10.1007/s40139-014-0052-0.

19) Pedersen IM, Cheng G, Wieland S. Interferon modulation of cellular microRNAs as an antiviral mechanism. Nature. 2007; 449: 919-22. doi: 10.1038/nature06205, PMID: 17943132, PMCID: PMC2748825.

20) Shrivastava Sh, Steele R, Ray R, Ray RB. MicroRNAs: Role in hepatitis C virus pathogenesis. Genes and Diseases. 2015; 2(1): 35-45. doi: 10.1016/j.gendis.2015.01.001.

21) Sarasin-Filipowicz M, Krol J, Markiewicz I, Heim MH, Filipowicz W. Decreased levels of micro RNA miR-122 in individuals with hepatitis C responding poorly to interferon therapy. Nat Med. 2009; 15: 31 -33. doi: 10.1038/nm.1902, PMID: 19122656.

22) Shrivastava Sh, Mukherjee A, Ray R. hepatitis C virus infection, microRNA and liver disease progression. World J Hepatol. 2013; 5(9): 479-86. PMID: 24073299, PMCID: PMC3782685.

23) Nasheri N, Singaravelu R, Goodmurphy M, Lyn PK, Pezacki JP. Competing roles of micro RNA-122 recognition elements in hepatitis $\mathrm{C}$ virus RNA. Virology. 2011; 410: 336-44. doi: 10.1016/j.virol.2010.11.015, PMID: 21185047. 